\title{
Primordial gravitational waves in running vacuum cosmologies
}

\author{
D.A. Tamayo ${ }^{a}$, J.A.S. Lima ${ }^{\mathrm{b}}$, M.E.S. Alves ${ }^{\mathrm{c}}$, J.C.N. de Araujo ${ }^{\mathrm{d}, *}$ \\ a Instituto de Física, Universidade de São Paulo, Rua do Matão, 05508-090, São Paulo, SP, Brazil

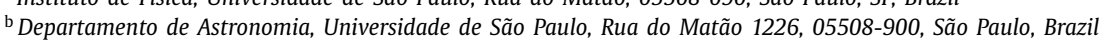 \\ ${ }^{\mathrm{C}}$ Instituto de Ciência e Tecnologia, Universidade Estadual Paulista, São José dos Campos, SP, 12247-016, Brazil \\ ${ }^{\mathrm{d}}$ Divisão de Astrofísica, Instituto Nacional de Pesquisas Espaciais, Avenida dos Astronautas 1758, São José dos Campos, 12227-010 SP, Brazil
}

\section{A R T I C L E I N F O}

\section{Article history:}

Received 26 August 2015

Revised 6 June 2016

Accepted 20 November 2016

Available online 23 November 2016

\section{Keywords:}

Gravitational waves

Wave generation and sources

Relativity and gravitation

Cosmology

\begin{abstract}
A B S T R A C T
We investigate the cosmological production of gravitational waves in a nonsingular flat cosmology powered by a "running vacuum" energy density described by $\rho_{\Lambda} \equiv \rho_{\Lambda}(H)$, a phenomenological expression potentially linked with the renormalization group approach in quantum field theory in curved spacetimes. The model can be interpreted as a particular case of the class recently discussed by Perico et al. (2013) [25] which is termed complete in the sense that the cosmic evolution occurs between two extreme de Sitter stages (early and late time de Sitter phases). The gravitational wave equation is derived and its time-dependent part numerically integrated since the primordial de Sitter stage. The generated spectrum of gravitons is also compared with the standard calculations where an abrupt transition, from the early de Sitter to the radiation phase, is usually assumed. It is found that the stochastic background of gravitons is very similar to the one predicted by the cosmic concordance model plus inflation except at higher frequencies $(v \gtrsim 100 \mathrm{kHz}$ ). This remarkable signature of a "running vacuum" cosmology combined with the proposed high frequency gravitational wave detectors and measurements of the CMB polarization (B-modes) may provide a new window to confront more conventional models of inflation.
\end{abstract}

(c) 2016 Elsevier B.V. All rights reserved.

\section{Introduction}

In the last decade, many authors have proposed cosmological models driven by a "running vacuum" energy density, $\rho_{\mathrm{vac}}=$ $\Lambda(H) / 8 \pi G$. The leitmotiv of such an idea is related to two basic difficulties of the standard $\Lambda \mathrm{CDM}$ model (constant vacuum energy density). Firstly, a dynamical $\Lambda(H)$-term may solve the so-called cosmological constant problem because in this case the vacuum energy density relaxes to its present value and one may argue that $\Lambda$ is small today because the expanding Universe is too old. Secondly, a dynamical- $\Lambda(H)$ term may also solve the so-called cosmic coincidence problem, i.e. the fact that the time-varying matter energy density and the (constant) vacuum energy density have the same order of magnitude nowadays.

Since long ago, many different phenomenological decay laws for $\Lambda(H)$ were proposed in the literature (see [1-5] for the oldest literature and [6-9] for more recent articles). The predictions of the latest models have also been confronted with the available obser-

\footnotetext{
* Corresponding author. Fax: +551232086811.

E-mail addresses: tamayo@if.usp.br (D.A. Tamayo), jas.lima@iag.usp.br (J.A.S. Lima), marcio.alves@ict.unesp.br (M.E.S. Alves), jcarlos.dearaujo@inpe.br (J.C.N. de Araujo).
} http://dx.doi.org/10.1016/j.astropartphys.2016.11.007 0927-6505/C 2016 Elsevier B.V. All rights reserved. vational data and the results compared with the predictions of the standard $\Lambda$ CDM cosmology [10,11].

Besides enlarging the standard view of a rigid $\Lambda$-term, there are also some attempts to justify theoretically a "running vacuum" cosmology based on different methods, among them: the thermal instability of a de Sitter spacetime [12] and the renormalization group (RG) approach in curved spacetimes [13]. In the latter case, for instance, the emerging dynamical $\Lambda(H)$-term (beyond the true constant vacuum contribution) depends on an expansion power series on the Hubble parameter $\left(\Sigma H^{n}\right)$ where only the even powers of $\mathrm{H}$ are involved in the RG realization, selected by the general covariance of the effective action appearing in the quantum field theoretical treatment in curved spacetimes [13-15]. In the same vein, several alternative approaches also try to represent the interacting $\Lambda(H)$ models through a Lagrangian description as in the $F(T)$ gravity approach [16], or in its generalized form $F(R, T)$, as discussed by Harko and coworkers [17]. Other attempts involve a mixture of a scalar field interacting with the radiation bath $[18,19]$, as in the socalled warm inflationary model [20-23], or still based on the quantum mechanics probability of unstable states [24]. Although interesting and highly promising to understand the decaying vacuum problem in the evolving Universe, none of them can at present be considered definitive and/or widely accepted by the community. 
In this connection, a large class of nonsingular models, where the vacuum energy density evolves phenomenologically as a truncated power-series in the Hubble parameter, has been proposed a couple of years ago [25,26] (its dominant term $\rho_{\mathrm{vac}}(H) \propto H^{n}, n$ $>2$ ). This class of models has some interesting features, among them: a nonsingular origin for the expanding universe (no horizon problem) with a deflationary process also without "exit problem", that is, the model evolves smoothly from the primeval nonsingular de Sitter state to the radiation phase; its late-time cosmic expansion history is very close to the concordance model and it also furnishes a smooth link between the initial and final de Sitter stages through the radiation and matter dominated phases. The temperature behavior and the entropy generation during the continuous non-adiabatic transition from de Sitter to the radiation phase has also been investigated $[27,28]$ and a comparison with the late time observations has also been carried out in detail by Gomez-Valent and Solà [29].

Furthermore, the recent LIGO detection of gravitational waves (GWs) opened a new window of observations for astronomy and cosmological problems [30]. Its importance goes much far beyond the search for signatures of compact binary coalescence (black holes, neutron stars, etc). For instance, tensor perturbations describing GWs generated in the inflationary stage may affect the pattern of Cosmic Background Radiation (CMB) anisotropies through the B-modes polarization [31]. In addition, there are also ongoing projects (BICEP3, Keck Array experiments, SPT) and future probes with high sensitivity instruments like the QUBIC, especially designed to measure the B-modes with high precision $[32,33]$. Naturally, as compared with more conventional inflationary models, the amplification of GWs from a primordial de Sitter stage supported by a decaying- $\Lambda(H)$ model may affect CMB polarization in a different way, and, as such, this line of inquire deserves a closer scrutiny.

In this context, we analyze here the production of primordial GWs for a class of nonsingular "running vacuum cosmologies". The phenomenological $\Lambda(H)$-term adopted here is defined by: $\Lambda(H)=$ $\Lambda_{b}+\alpha H^{3} / H_{I}$, where $\Lambda_{b}$ is the constant bare vacuum energy density, $H$ is the Hubble parameter, $H_{I}$ is the primordial inflationary scale and $\alpha$ is a dimensionless free parameter. Therefore, unlike the general class discussed in Refs. [25,26], the very late time behavior of the model analyzed here is exactly $\Lambda \mathrm{CDM}$. The GW equation is derived and its time-dependent part numerically integrated since the primordial de Sitter stage.

As we shall see, for higher frequencies $(v \geq 100 \mathrm{kHz})$ the predicted spectrum departs from the standard inflationary prediction, and, as such, these models are distinguishable if high frequency GW detectors become operative and reach the expected sensitivity in the near future. In general grounds, this signal reinforces the possibility of a new observational approach to inflationary physics, and provides additional motivation in the search for stochastic background of GWs at high frequencies [34-36]). It is also argued that similar results remain valid for generic decaying vacuum cosmologies with an initial de Sitter stage supported by a power-law $H^{n}$ with $n>2$.

\section{The model: basic equations}

Let us now consider that the Universe is described by a flat Friedmann-Lemâitre-Robertson-Walker (FLRW) geometry. In the co-moving coordinate system, the background line element reads $(c=1)$ :

$d s^{2}=d t^{2}-a^{2}(t) d l^{2}$

where $a(t)$ is the scale factor.

The Einstein equations in the above background read

$8 \pi G \rho+\Lambda(t)=3 H^{2}$
$8 \pi G p-\Lambda(t)=-2 \dot{H}-3 H^{2}$,

where a dot means time derivative and $H=\dot{a} / a$ is the Hubble parameter. To solve the above set of equations one needs the functional form of $\Lambda(t)$, or equivalently, $\Lambda(H)$, as well as an equation of state (EoS).

As remarked before, it will be assumed here that the $\Lambda(H)$-term is given by a particular case of the general class studied by Perico et al. $[25,26]$ (for closely related works see also $[3,4]$ )

$8 \pi G \rho_{\mathrm{vac}}(H)=\Lambda(H)=\Lambda_{b}+3 \alpha \frac{H^{3}}{H_{I}}$,

where $\Lambda_{b}$ is the bare cosmological constant. By assuming that the material medium obeys the EoS, $p=\omega \rho$, where $\omega$ is a different constant for each era, one may show that the scale factor and the Hubble parameter obey the following equations (from now on, without loss of generality we consider $\alpha=1$ ):

$2 \dot{H}+3(1+\omega) H^{2}\left[1-\frac{H}{H_{I}}\right]-(1+\omega) \Lambda_{b}=0$.

The standard cosmic concordance model equations are recovered by taking the limits $H_{I} \gg H$ and $\omega=0(\Lambda \mathrm{CDM})$. At early times, the last term proportional to the bare $\Lambda_{b}$ can safely be neglected. In this case, for $(\omega=1 / 3)$ the solution of the above equation takes the following form:

$H=\frac{H_{I}}{1+C a^{2}}$,

where $C$ is an integration constant. We see that the transition from an early de Sitter $\left(\mathrm{Ca}^{2} \ll 1, H \sim \mathrm{H}_{I}\right)$ to the radiation phase $\left(\mathrm{Ca}^{2} \gg\right.$ $\left.1, a \propto t^{1 / 2}\right)$ is analytically described.

Now, in terms of the conformal time $[d t=a(\eta) d \eta]$, the line element (1) becomes:

$d s^{2}=a^{2}(\eta)\left[d \eta^{2}-\delta_{i j} d x^{i} d x^{j}\right]$,

while the equation of motion (5) takes the form

$2 \frac{H^{\prime}}{a}+3(1+\omega) H^{2}\left[1-\frac{H}{H_{I}}\right]-(1+\omega) \Lambda_{b}=0$,

where primes denote derivative with respect to $\eta$. By fixing the EoS parameters, the integration constants for each era are obtained by imposing the continuity conditions for $a(\eta)$ and $a^{\prime}(\eta)$ at the transition times between two subsequent eras.

By assuming that the vacuum decay mainly into ultrarelativistic particles $(\omega=1 / 3)$ when the bare term of the decaying vacuum is negligible $\left(\Lambda_{b} \ll H^{3} / H_{I}\right)$, it is easy to see that (8) boils down to $a^{\prime \prime}-\frac{2}{H_{I}}\left(\frac{a^{\prime}}{a}\right)^{3}=0$. A direct integration of this equation yields the following solution:

$a(\eta)=\frac{1}{2 C_{1}}\left[\eta+C_{2}+\sqrt{\left(\eta+C_{2}\right)^{2}+\frac{4 C_{1}}{H_{I}}}\right]$,

which has two limiting cases: at very early times it is a de Sitter solution, $a \propto|\eta|^{-1}$, whereas at late time $\left(t>>H_{I}^{-1}\right)$, it enters in the radiation phase, $a \propto \eta[25,28]$. The reduced Hubble parameter, $\mathcal{H}(\eta)=a^{\prime} / a$, for this stage

$\mathcal{H}_{\text {Inf-R }}(\eta)=\frac{a^{\prime}}{a}=\left[\left(\eta+C_{2}\right)^{2}+\frac{4 C_{1}}{H_{I}}\right]^{-1 / 2}$,

assumes its maximum value for $\eta=-C_{2}$, so $\mathcal{H}\left(-C_{2}\right)=\mathcal{H}\left(\eta_{\max }\right) \equiv$ $\mathcal{H}_{\max }=\sqrt{\frac{H_{I}}{4 C_{1}}}$. Therefore, we can rewrite the integrating constants in the form $C_{1}=4 H_{I}^{-1} \mathcal{H}_{\max }^{2}$ and $C_{2}=-\eta_{\max }$, and, naturally, (10) can be rewritten in terms of the new pair of constants $\left(\eta_{\max }, \mathcal{H}_{\max }\right)$, a form that will be useful in the next section. 

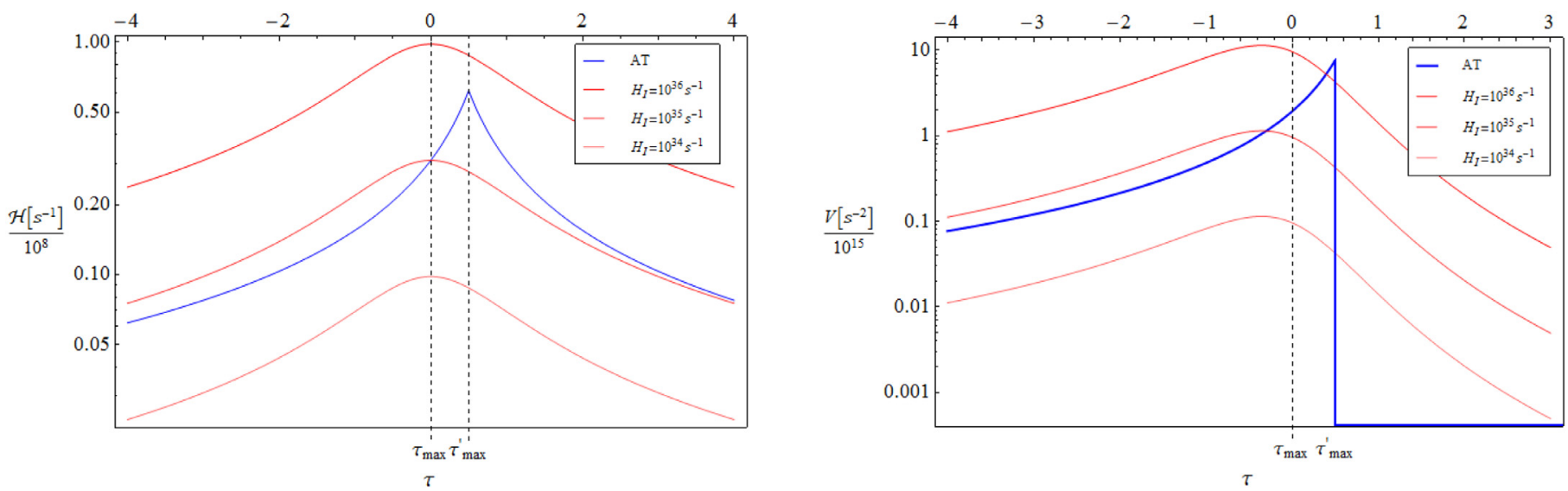

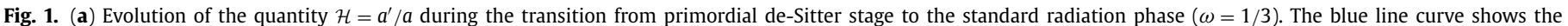

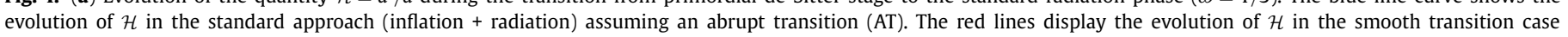

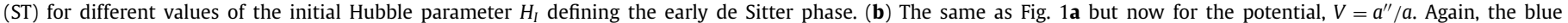

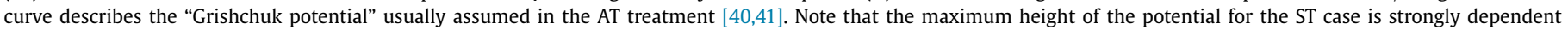

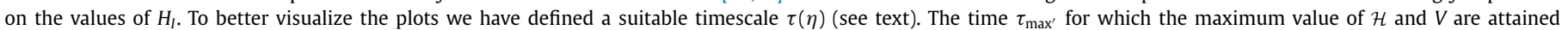

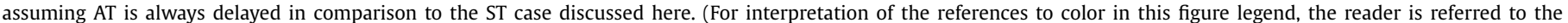
web version of this article.)

\section{Cosmological tensor perturbations}

The classical tensor metric perturbation for the conformal FLRW flat metric (7) can be written as [39]:

$d s^{2}=a^{2}(\eta)\left[d \eta^{2}-\left(\delta_{i j}+h_{i j}\right) d x^{i} d x^{j}\right]$,

where the perturbation $h_{i j}$ is small, $\left|h_{i j}\right| \ll 1$, is transverse-traceless and satisfy the gauge constraints: $h_{0 \mu}=0, h_{i}^{i}=0, \nabla^{j} h_{i j}=0$.

The first order evolution equation of $h_{i}^{j}$ is given by [37-39]

$h_{i}^{j^{\prime \prime}}+2 \frac{a^{\prime}}{a} h_{i}^{j^{\prime}}-\nabla^{2} h_{i}^{j}=16 \pi G a^{2} \delta \bar{T}_{j(T)}^{i}=0$,

where in the last equality we used that $\delta \bar{T}_{j(T)}^{i} \equiv 0$ because the total EMT (matter plus vacuum) has the perfect fluid isotropic form [39]. The general solution of the above equation can be Fourier expanded in the standard way

$$
\begin{aligned}
h_{i j}(\eta, \mathbf{x})= & \frac{\sqrt{16 \pi G}}{(2 \pi)^{3 / 2}} \int d^{3} \mathbf{n} \sum_{r=+, \times} \stackrel{r}{\epsilon}(\mathbf{n}) \\
& \times\left[\begin{array}{c}
r \\
h_{n}(\eta) e^{i \mathbf{n} \cdot \mathbf{x}}
\end{array}{ }_{C_{\mathbf{n}}^{r}}^{r}+h_{n}^{*}(\eta) e^{-i \mathbf{n} \cdot \mathbf{x}}{ }_{c_{\mathbf{n}}^{r}}^{\dagger}\right],
\end{aligned}
$$

where ${ }_{h} h_{n}(\eta)$ are the mode functions, $\mathbf{n}$ is the comoving wave vector, $\stackrel{r}{c_{\mathbf{n}}}$ and ${\stackrel{r}{c_{\mathbf{n}}}}^{\dagger}$ are complex numbers, and $\stackrel{r}{\epsilon}$ ij $(\mathbf{n})$ is the symmetric, transverse-traceless polarization tensor. Now, by recalling that the comoving wave number is given by

$n=|\mathbf{n}|=\frac{2 \pi a(\eta)}{\lambda}=k a(\eta)$,

and inserting the solution (13) into (12), it is readily seen that the temporal part yields a differential equation valid for both polarizations (henceforth we drop the index $r$ )

$h_{n}(\eta)^{\prime \prime}+2 \mathcal{H}(\eta) h_{n}(\eta)^{\prime}+n^{2} h_{n}(\eta)=0$.

where we have conveniently used the reduced Hubble parameter, $\mathcal{H}$, whose continuity also guarantees the continuity of the first derivative of the scale factor. By using the auxiliary function, $\mu=h_{n}(\eta) a(\eta)$, the above equation assumes the form first derived by Grishchuck $[40,41]$ :

$\mu^{\prime \prime}+\left(n^{2}-\frac{a^{\prime \prime}}{a}\right) \mu=0$,
This is the basic equation which allow us to obtain the associated physical quantities like the wave amplitude, energy density and power spectrum.

The scale of the "Grishchuck potential", $V(\eta)=a^{\prime \prime} / a$, as compared with the wave-number determines the behavior of the limiting solutions for $\mu(\eta)$. The solutions are oscillatory, $\mu \propto e^{ \pm i n \eta}$, when $n^{2} \gg|V|$ holds. The high-frequency modes in this case are diluted by the cosmic expansion since $h=e^{ \pm i n \eta} / a$. In the opposite regime one finds $\mu \propto a$ so that the amplitude remains constant. In this case, the damping of the waves due the universe expansion is avoided, a phenomenon usually referred to as adiabatic amplification $[40,41]$.

In Fig. $1 \mathrm{a}$ and $\mathrm{b}$ we show the behavior of the quantities $\mathcal{H}(\eta)$ and $V(\eta)$ during the transition from the early de Sitter stage to the radiation phase. For the sake of clarity, both quantities were expressed in terms of a convenient variable, $\tau=\mathcal{H}_{\max }\left(\eta_{\max }-\eta\right)$, where $\eta_{\max }$ is the time when $\mathcal{H}=\mathcal{H}_{\max }$ is reached. As shown below Eq. (18), the value of $\mathcal{H}_{\max }$ depends on the scale $H_{I}$. In the variable $\tau$, the maximum of $\mathcal{H}$ for the decaying vacuum model occurs at $\tau_{\max }=0$ while for the abrupt case the maximum is delayed $\left(\tau_{\max }^{\prime}>\tau_{\max }\right)$. The interesting point here is that such a transition in our model is smooth and can analytically be followed in terms of the scale factor and the quantity $\mathcal{H}$.

\section{Gravitational wave solutions}

Let us now determine the generated spectrum of the GWs. During the transition from inflation to radiation (Inf-R), the GW Eq. (16) assumes the form

$\mu_{\text {Inf-R }}^{\prime \prime}+\left(n^{2}-V_{\text {Inf-R }}(\eta)\right) \mu_{\text {Inf-R }}=0$,

where $V_{\text {Inf-R }}(\eta)=a^{\prime \prime} / a \equiv \mathcal{H}_{\text {Inf-R }}^{2}+\mathcal{H}_{\text {Inf-R }}^{\prime}$. From Section 2 one finds that the quantity $\mathcal{H}_{\text {Inf-R }}$ for $\omega=1 / 3$ reads:

$\mathcal{H}_{\text {Inf-R }}(\eta)=\frac{1}{\sqrt{\left(\eta-\eta_{\max }\right)^{2}+1 / \mathcal{H}_{\max }^{2}}}$,

where we have at $\eta_{\max }$ the maximum value $\mathcal{H}_{\max } \simeq H_{0} \sqrt{\eta_{\mathrm{eq}} H_{I} / 8}$ and $\eta_{\text {eq }}$ is the conformal time of equality of the energy densities of radiation and matter. Since the scenario starts as a de Sitter spacetime (see discussion below Eq. (6)), it is possible to obtain an analytical solution for $\mu_{\text {Inf }}$ at that time. In addition, by imposing the 

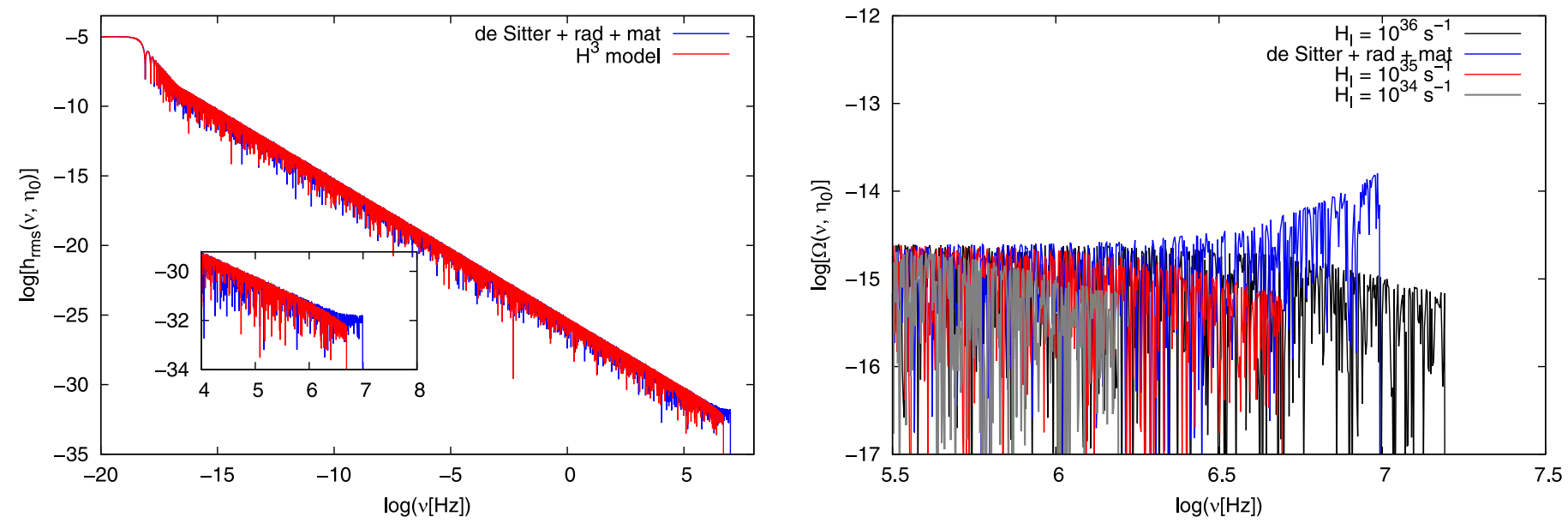

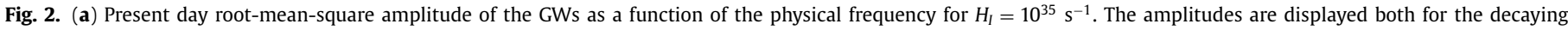

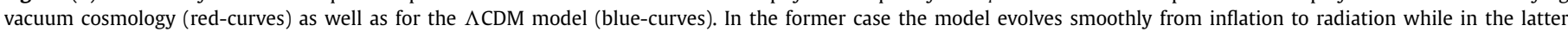

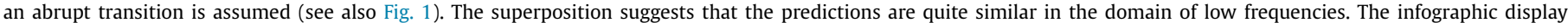

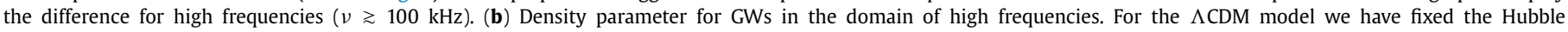

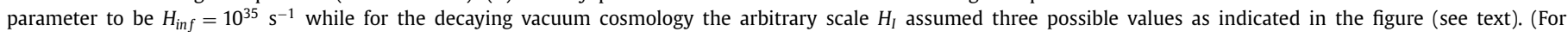
interpretation of the references to color in this figure legend, the reader is referred to the web version of this article.)

adiabatic vacuum $\lim _{n \rightarrow \infty} \mu_{\text {Inf }} \propto e^{-i n \eta} / \sqrt{n}$ constraint [27], we find:

$\mu_{\text {Inf }}(n, \eta) \simeq \frac{A_{0}}{\sqrt{2 n}}\left(1-\frac{i}{n\left(\eta-\eta_{\max }\right)}\right) e^{-i n\left(\eta-\eta_{\max }\right)}$.

where $A_{0}$ is a real constant which specifies the initial amplitude of the GWs. The above expression for $\mu_{\text {Inf }}(n, \eta)$, as well as its derivative $\mu_{\text {Inf }}^{\prime}(n, \eta)$, provide the initial conditions (taken at the initial unstable de Sitter era) for the numerical integration of Eq. (17). Consequently, the evolution of the GWs can be traced until a time $\eta_{\mathrm{r}}$ from which the scale factor and waveforms behave as in the usual $\Lambda$ CDM scenario. At the radiation era, it is possible to find an analytical solution for $\mu(\eta)$ whose initial conditions (evaluated at $\eta_{\mathrm{r}}$ ) are given by the previous numerical integration. This solution is valid until the conformal time $\eta_{\mathrm{eq}}$, for which matter $(\omega=0)$ starts to dominate, and the scale factor evolves exactly as in the standard matter dominated cosmological scenario. At this stage, the analytical solution was obtained by considering the initial conditions (at $\eta_{\text {eq }}$ ) given at the end of the precedent radiation era. Finally, we have evaluated $\mu\left(\eta_{0}\right)$ at the present time $\eta_{0}$. In addition, from the standard definition, we also find the resulting present power spectrum of the relic GWs in this model:

$\mathcal{P}\left(n, \eta_{0}\right)=\frac{16 \ell_{\mathrm{Pl}}^{2}}{\pi} n^{3}\left|h_{n}\left(\eta_{0}\right)\right|^{2}$.

In Fig. 2a we show the present day root-mean-square (rms) amplitude of the GWs as a function of the physical frequency $v$ for the decaying vacuum model, which is related to the power spectrum via $h_{r m s}\left(\nu, \eta_{0}\right)=\sqrt{\mathcal{P}\left(\nu, \eta_{0}\right)}$. For comparison, we also show the rms amplitude for the abrupt three phase transition model (de Sitter, radiation and matter) with $H_{I}=10^{35} \mathrm{~s}^{-1}$. For simplicity, we have not included a late time $\Lambda_{b}$ dominated epoch since the same effect for both models is obtained, namely, a little smaller value of $h_{\text {rms }}$ for the complete GW spectrum [42].

Notice the remarkable superposition of both spectra for almost the entire spectral range. The models are distinguishable only at very high frequencies, which are displayed in detail in Fig. $2 b$, where we show the spectrum of the density parameter $\Omega_{\mathrm{GW}}(v$, $\eta_{0}$ ). In this figure, we have fixed the value of $H_{I}$ for the abrupt transition model and considered some possible values of $H_{I}$ for the decaying vacuum cosmology.

At high frequencies the two models predict distinct spectra for a given value of $H_{I}$. As shown in Fig. 1, such an effect can be un- derstood in terms of the behavior of $\mathcal{H}(\eta)$ and $V(\eta)$. Since the decaying vacuum model evolves smoothly from a de Sitter towards a radiation era, the shape of $\mathcal{H}(\eta)$ for the transition and the consequent lower value of $\mathcal{H}_{\max }$ (for the same $H_{I}$ ) result in a lower high frequency GW production.

On the other hand, there is no adiabatic amplification for frequencies $v>\mathcal{H}_{\max }$ so that we have introduced a cut-off at $v_{\max }=$ $\mathcal{H}_{\max }$ in these graphs. For a given $H_{I}$ value, the cut-off frequency for the abrupt transition model is twice the cut-off frequency for the decaying vacuum model. However, since we have freedom to choose $H_{I}$ for the $\Lambda(\mathrm{H})$-model, different cut-off frequencies are allowed (see Fig. 2b). In principle, even for different values of $H_{I}$, the shape of the curves predicted by the AT and ST models are quite different thereby suggesting a possible test for the underlying inflationary mechanisms.

\section{Final comments}

We have investigated the production of GWs in the context of a flat nonsingular decaying vacuum cosmology. The dynamical $\Lambda(\mathrm{H})$-term was phenomenologically modeled as $\Lambda(H)=\Lambda_{b}+$ $H^{3} / H_{I}$. This kind of model undergone a smooth transition from an early inflation to the standard radiation phase which can analytically be described $[3,4,25,26]$. Interestingly, the model explain the present day entropy content of the Universe stored in the relic radiation $[27,28]$ without necessity of a highly non-adiabatic reheating stage. Such scenario is also free of horizon and "graceful" exit problems, and is also in agreement with the observations at low and intermediate redshifts since it evolves to the standard $\Lambda_{b} \mathrm{CDM}$ cosmology. In comparison with the standard approach (big-bang, adiabatic inflation, reheating, plus radiation and subsequent eras), the present study also provides a simple way to understand how a different scenario can modify the predictions concerning the generated spectrum of GWs.

Solutions for the GW equation were numerically obtained. The result furnishes a simple and definite example that details of the transition from an early de Sitter to the radiation phase plays an important role in the generation of the GW spectrum. The identified lower GW production for high frequencies $(v \gtrsim 100 \mathrm{kHz})$ is a remarkable signature of the $\Lambda$ decay model (see Figs. $1 \mathrm{~b}$ and $2 \mathrm{~b}$ ). Although far exceeding the frequencies (and sensitivity) of the existing GW detectors in ground-based experiments and space-based 
successors like LISA, new kinds of interferometers and detectors operating in a frequency range high enough to test primordial GW have been discussed in the recent literature [43] (see also $[44,45]$ ). The high frequency behavior shows that the model is not only distinguishable from abrupt inflationary scenario but can also provide a crucial test for the underlying mechanism as predicted in some multi-field inflationary models [35]. Note also that current GW interferometers can not detect the putative GW background studied here, since the amplitude of this background is orders of magnitude lower than the interferometer sensitivities. We refer the reader to the paper by Moore, Cole and Berry [46], where the sensitivity curves of several detectors are displayed. Finally, it should be reinforced that the model discussed here is a starting point for the investigation of more complex and rich decaying vacuum cosmologies, like the general class proposed in Ref. $[25,26]$. Such models with $\Lambda(H) \propto H^{n}, n>2$ deserves a closer scrutiny since they furnish a complete cosmological history. In principle, although the details might be dependent on the power $n$, similar results to the ones derived here should be expected because the entire class also evolves through a smooth transition from de Sitter to the radiation era. A detailed analysis involving the generation of GWs in this more general framework plus the implications in the observed pattern of $\mathrm{CMB}$ anisotropies through the B-modes polarization will be discussed in a forthcoming communication.

\section{Acknowledgments}

DT is supported by a fellowship from CAPES. JASL and JCNA would like to thank CNPq, FAPESP and CAPES (PROCAD 2013) for partial financial support. We would also like to thank the referees for their helpful comments and suggestions.

\section{References}

[1] M. Ozer, M.O. Taha, Phys. Lett. B 171 (1986) 363.

[2] J.C. Carvalho, J.A.S. Lima, I. Waga, Phys. Rev. D 46 (1992) 2404.

[3] J.A.S. Lima, J.M.F. Maia, Phys. Rev. D 49 (1994) 5597.

[4] J.A.S. Lima, M. Trodden, Phys. Rev. D 53 (1996) 4280. astro-ph/9508049.

[5] J.M. Overduin, F.I. Cooperstock, Phys. Rev. D 58 (1998) 043506.
[6] S. Carneiro, J.A.S. Lima, Int. J. Mod. Phys. A 20 (2005) 2465. gr-qc/0405141.

[7] J.S. Alcaniz, J.A.S. Lima, Phys. Rev. D 72 (2005) 063516. astro-ph/0507372.

[8] H. Borges, S. Carneiro, J. Fabris, C. Pigozzo, Phys. Rev. D 77 (2008) 043513.

[9] F.E.M. Costa, J.A.S. Lima, F.A. Oliveira, Class. Quantum Gravity 31 (2014) 045004.

[10] S. Basilakos, Mon. Not. R. Astron. Soc. 395 (2009) 234.

[11] S. Basilakos, D. Polarski, J. Solà, Phys. Rev. D 86 (2012) 043010.

[12] M. Gasperini, Phys. Lett. B 194 (1987) 347.

[13] J. Solà, J. Phys. Conf. Ser. 283 (2011) 012033.

[14] I.L. Shapiro, J. Solà, Phys. Lett. B 475 (2000) 236.

[15] I.L. Shapiro, J. Solà, J. High Energy Phys. 02 (2002) 006. hep-th/0012227.

[16] N.J. Poplawski, ArXiv:gr-qc/0608031v2.

[17] T. Harko, F.S.N. Lobo, S. Nojiri, S.D. Odintsov, Phys.Rev. D 84 (2011) 024020. 1104.2669.

[18] J.M.F. Maia, J.A.S. Lima, Phys. Rev. D 65 (2002) 083513. astro-ph/0112091.

[19] D.F.A. Bessada, Phys. Rev. D 88 (2013) 023005. 1307.1099[gr-qc].

[20] A. Berera, L.Z. Fang, Phys. Rev. Lett. 74 (1995) 1912.

[21] A. Berera, Phys. Rev. Lett. 75 (1995) 3218.

[22] A. Berera, I. Moss, R. Ramos, Rept. Prog. Phys. 72 (2009) 026901.

[23] J.M.F. Maia, J.A.S. Lima, Phys. Rev. D 60 (1999) 101301. astro-ph/9910568.

[24] M. Szydlowski, Phys. Rev. D 91 (2015) 123538.

[25] E.L.D. Perico, J.A.S. Lima, S. Basilakos, J. Solà, Phys. Rev. D88 (2013) 063531. 1306.0591[astro-ph.CO].

[26] J.A.S. Lima, S. Basilakos, J. Solá, Mon. Not. R. Astron. Soc. 431 (2013) 923. 1209. 2802[gr-qc].

[27] J.A.S. Lima, S. Basilakos, J. Solá, Gen. Rel. Grav. 47 (2015) 40. 1412.5196[gr-qc].

[28] J.A.S. Lima, S. Basilakos, J. Solà, Eur. Phys. J. C76 (2016) 228. 1509.00163v2.

29] A. Gomez-Valent, J. Solà, Mon. Not. R. Astron. Soc. 448 (2015) 2810.

[30] B. Abbott, et al., Phys. Rev. Lett. 116 (2016) 061102.

[31] P.A.R. Ade(planck collaboration), XIII. cosmological parameters, 2015. 1502 01589[astro-ph.CO].

[32] E. Battisteli, et al., The QUBIC collaboration 2010 (2010). 10100645.

[33] A. Ghrib, et al., Latest progress on the QUBIC instrument, J. Low Temp. Phys. 176 (2014) 698.

[34] H. Tashiro, T. Chiba, M. Sasaki, Class. Quant. Grav. 21 (2004) 1761.

[35] R. Easther, E.A. Lim, J. Cosmol. Astropart. Phys. 04 (2006) 010.

[36] J. Garcia-Bellido, D.G. Figueroa, A. Sastre, Phys. Rev. D 77 (2008) 043517.

[37] M.R. de Garcia Maia, J.A.S. Lima, Phys. Rev. D 54 (1996) 6111.

[38] D.A. Tamayo, J.A.S. Lima, D. Bessada, ArXiv:1503.06110[astro-ph.CO].

[39] V. Mukhanov, Physical Foundations of Cosmology, Cambridge University Press, 2010.

[40] L.P. Grishchuk, J. Exp. Theor. Phys. 40 (1975) 409.

[41] L.P. Grishchuk, Class. Quant. Grav. 10 (1993) 2449.

[42] H.X. Miao, Y. Zhang, Phys. Rev. D75 (2007) 104009.

[43] T. Akutsu, et al., Phys. Rev. Lett. 101 (2008) 101101. 0803.4094[gr-qc].

[44] A. Arvanitaki, A.A. Geraci, Phys. Rev. Lett. 110 (2013) 071105.

[45] M. Goryachev, M.E. Tobar, Phys. Rev. D 90 (2014) 102005

[46] C.J. Moore, R.H. Cole, C.P.L. Berry, Class. Quant. Grav. 32 (2015) 015014. 